CrossMark \&click for updates

Cite this: Med. Chem. Commun., 2016, 7, 190

Received 4th September 2015, Accepted 10th November 2015

DOI: $10.1039 / \mathrm{c} 5 \mathrm{md} 00380 \mathrm{f}$

www.rsc.org/medchemcomm

\title{
In vitro and in vivo activity of ML302F: a thioenolate inhibitor of VIM-subfamily metallo $\beta$-lactamases
}

\author{
Jonathan W. Betts, ${ }^{a}$ Lynette M. Phee, ${ }^{\text {ab }}$ Muhd H. F. Abdul Momin, ${ }^{a}$ \\ Klaus-Daniel Umland, ${ }^{C}$ Jurgen Brem, ${ }^{C}$ Christopher J. Schofield ${ }^{C}$ \\ and David W. Wareham*ab
}

\begin{abstract}
The thioenol ML302F was recently identified as an inhibitor of class B metallo- $\beta$-lactamases (MBLs). We assessed the activity of ML302F when combined with meropenem (MEM) against 31 carbapenem resistant Gram-negative clinical isolates. Minimum inhibitory concentrations of MEM: ML302F were determined at fixed ratios of $1: 4$ and $1: 8$ using strains producing variants of the clinically relevant VIM-like MBL. Toxicity and efficacy in vivo was assessed in a Galleria mellonella invertebrate model against strains producing VIM1, VIM-2 and VIM-4 variants. At a fixed MEM : ML302F ratio of $1: 8,22 / 31$ isolates were rendered either susceptible ( $\mathrm{MIC} \leq 2 \mathrm{mg} \mathrm{L}^{-1}$ ), or intermediate (MIC 4-8 $\mathrm{mg} \mathrm{L}^{-1}$ ) to MEM. ML302F alone was not toxic at up to $80 \mathrm{mg} \mathrm{kg}^{-1}$ in G. mellonella and treatment with MEM $0.6 \mathrm{mg} \mathrm{kg}^{-1}:$ ML302F $4.8 \mathrm{mg} \mathrm{kg}^{-1}$ significantly improved the survival of infected larvae. As ML302F was able to successfully restore susceptibility to resistant strains both in vitro and in vivo it represents a structurally interesting inhibitor in the search for new MBL inhibitors.
\end{abstract}

\section{Introduction}

The global dissemination of multi-drug resistant Gramnegative bacteria has seriously limited antibiotic treatment options. As few novel synthetic antimicrobials are in development the challenge is to increase and protect the lifespan and spectrum of existing compounds for as long as possible. This is most important in the case of $\beta$-lactams (penicillins, cephalosporins, carbapenems) especially when resistance occurs due to the acquisition and production of $\beta$-lactamases that catalyse $\beta$-lactam hydrolysis. ${ }^{1}$ There are two distinct mechanistic classes of $\beta$-lactamase - the nucleophilic serine enzymes and the metallo $\beta$-lactamases (MBLs). Inhibitors of the serine enzymes (clavulanic acid/tazobactam/avibactam) have been developed and already applied clinically. ${ }^{2}$ However, no such compounds have yet been developed to target MBLs. The use of carbapenems such as ertapenem, imipenem or meropenem (MEM) in combination with an effective metallo- $\beta$-lactamase inhibitor, could prolong their lifespan as well as increase their spectrum of use to carbapenemase producing bacteria. Thus, there are ongoing efforts to develop MBL inhibitors of suitable potency and breadth of selectivity for clinical use. ${ }^{3}$ In the latter regard the ability to inhibit the

\footnotetext{
${ }^{a}$ Antimicrobial Research Group, Barts \& The London School of Medicine and Dentistry, Queen Mary University of London, London, E1 2AT, UK.

E-mail:d.w.wareham@qmul.ac.uk

${ }^{b}$ Division of Infection, Barts Health NHS Trust, London, E1 1BB, UK

${ }^{c}$ Department of Chemistry, University of Oxford, Oxford, OX1 3TA, UK
}

different subtypes of clinically relevant MBLs and apparently rapidly emerging variants will likely be important.

The rhodanine ML302 was initially identified as a MBL inhibitor in a high throughput screen and subsequently characterised as a non-competitive inhibitor of class B (IMP, VIM, NDM) metallo- $\beta$-lactamases in vitro. ${ }^{4}$ Recently the mechanism behind the apparent inhibition by ML302 was investigated in crystallographic, NMR, and kinetic studies. These, unexpectedly revealed that ML302 undergoes hydrolysis, to generate the thioenol ML302F (Fig. 1). The thiol of ML302F chelates the two zinc ions present in the active site of most MBLs in a manner mimicking an intermediate in $\beta$-lactam hydrolysis. ${ }^{5}$ In some cases the observed inhibition of MBLs by ML302/ML302F combinations may involve ternary complexes. In some instances ML302F is able to inhibit the carbapenemase activity of MBLs at low $\mu \mathrm{M}$ concentrations $\left(\mathrm{IC}_{50}\right.$ VIM-2 $\left.0.30 \mu \mathrm{M}\right)$ sufficient to restore the activity of meropenem against MBL-producing strains. ${ }^{5}$

Neither ML302 nor ML302F have significant cytotoxicity towards eukaryotic cells in tissue culture $(>100 \mu \mathrm{M})$ but have not yet been assessed in any animal model of antimicrobial therapeutics. The wax moth caterpillar Galleria mellonella is increasingly being used as a simple invertebrate model for pre-clinical assessment of the efficacy, toxicity and pharmacokinetics of antimicrobials. ${ }^{6-9}$ Here we report studies on the activity of ML302F in combination with MEM against a collection of carbapenem resistant clinical isolates (Pseudomonas, Klebsiella, Enterobacter, Escherichia coli, Providencia) 
a)<smiles>O=C(O)c1cc(Cl)c(Cl)cc1Cl</smiles>
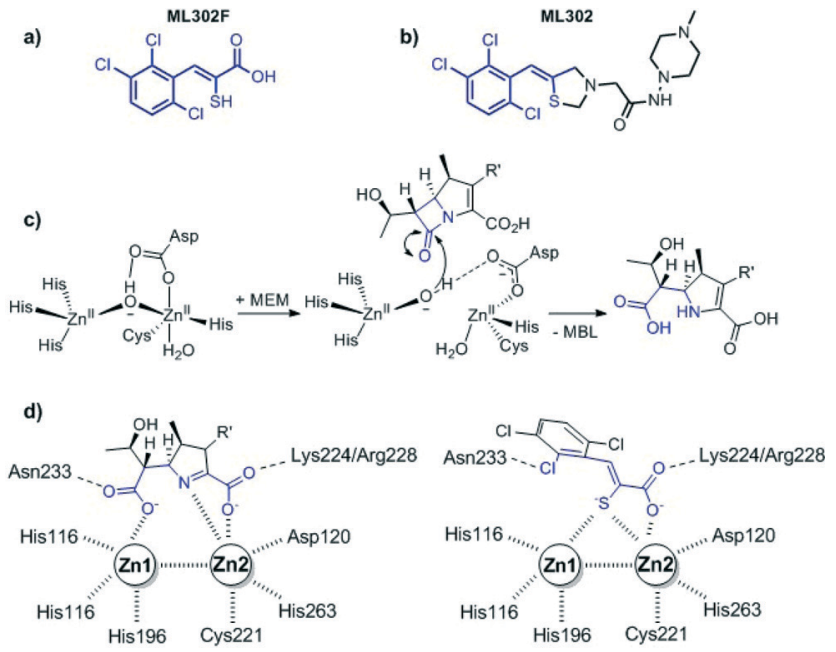

Fig. 1 Structures of (a) the thioenol ML302F, (b) the 'parent' rhodanine $M L 302$, (c) outline mechanism for MBL catalysed $\beta$-lactam hydrolysis as exemplified with meropenem (PDB ID: 4EYL), and (d) cartoons based on a crystal structure of ML302F (PDB ID: 4PVO) complexed with MBLs showing how the inhibitor mimics an enzymeintermediate/product complex.

producing clinically relevant variants of the VIM (VIM-1/2/4) MBL. Preferred ratios of MEM to ML302F required for synergy in vitro were determined and then used at a simulated humanized pharmacokinetic dose in the treatment of $G$. mellonella infected with carbapenem resistant $P$. aeruginosa, K. pneumoniae and E. coli.

\section{Results and discussion}

The antimicrobial activities of MEM and ML302F alone and in combination were assessed in vitro against 31 VIMproducing clinical isolates. Synergy between MEM and ML302F was first confirmed in checkerboard assays against 7/8 isolates tested (FICI $\leq 0.5$ ). An MEM : ML302F ratio of $1: 4$ and 1:8 was found to be optimal resulting in a $\geq 4$ fold reduction in the MIC of MEM.

Thirty strains were either resistant $\left(\mathrm{MIC}>8 \mathrm{mg} \mathrm{L}^{-1}\right)$ or had reduced susceptibility ( $\mathrm{MIC} \geq 4 \mathrm{mg} \mathrm{L}^{-1}$ ) to MEM alone as judged by the European Committee on Antimicrobial Susceptibility (EUCAST) breakpoint criteria. One $K$. pneumoniae isolate harbouring $b l a_{\mathrm{VIM}-1}$ was deemed susceptible (MIC 1 $\mathrm{mg} \mathrm{L}^{-1}$ ). ML302F had no antimicrobial activity alone at concentrations up to $512 \mathrm{mg} \mathrm{L}^{-1}$.

Using a fixed 1:8 ratio of MEM : ML302F in vitro susceptibility to carbapenems was increased in $22 / 31$ (70\%) of the isolates with 11 rendered susceptible (MIC $\leq 2 \mathrm{mg} \mathrm{L}^{-1}$ ) or intermediate (MIC $4-8 \mathrm{mg} \mathrm{L}^{-1}$ ). For 9 isolates, the MIC of MEM remained $>8 \mathrm{mg} \mathrm{L}^{-1}$ (Table 1 ).

ML302F was non-toxic when administered to G. mellonella by injection at doses up to $80 \mathrm{mg} \mathrm{kg}^{-1}$ (100\% survival of all larvae at $96 \mathrm{~h}$ ). The inoculum required for staggered killing of $>50 \%$ of larvae by carbapenem resistant strains over $96 \mathrm{~h}$ varied by species. The $\mathrm{LD}_{50}$ was $<10^{1}, 10^{5}$ and $10^{4} \mathrm{CFU}$ per
Table 1 Minimum inhibitory concentrations (MICs) of meropenem (MEM) with and without ML302F (1:8) versus multidrug-resistant Gramnegative bacteria producing VIM carbapenemases

\begin{tabular}{|c|c|c|c|c|}
\hline \multirow[b]{2}{*}{ MBL } & \multirow[b]{2}{*}{ Isolate } & \multicolumn{2}{|c|}{$\operatorname{MIC}\left(\mathrm{mg} \mathrm{L}^{-1}\right)$} & \multirow[b]{2}{*}{ FICI } \\
\hline & & MEM & MEM + ML302F & \\
\hline VIM-1 & K. pneumoniae GR54 & 128 & 4 & 0.09 \\
\hline VIM-1 & К. pneumoniae 177 & 1 & 1 & 1.02 \\
\hline VIM-1 & P. stuartii 67 & 8 & 1 & 0.14 \\
\hline VIM-1 & P. stuartii 70 & 8 & 1 & 0.14 \\
\hline VIM-2 & P. aeruginosa 30 & 64 & 8 & 0.25 \\
\hline VIM-2 & P. aeruginosa 47 & 32 & 1 & 0.05 \\
\hline VIM-2 & P. aeruginosa 50 & 32 & 8 & 0.38 \\
\hline VIM-2 & P. aeruginosa GR57 & 32 & 4 & 0.19 \\
\hline VIM-2 & P. aeruginosa GR58 & 32 & 8 & 0.38 \\
\hline VIM-2 & P. aeruginosa GR62 & 32 & 8 & 0.38 \\
\hline VIM-2 & P. aeruginosa GR64 & 32 & 4 & 0.19 \\
\hline VIM-2 & P. aeruginosa 3 (13) & 64 & 8 & 0.25 \\
\hline VIM-2 & P. aeruginosa 8 (13) & 128 & 16 & 0.38 \\
\hline VIM-2 & P. aeruginosa 9 (13) & 128 & 16 & 0.38 \\
\hline VIM-2 & P. aeruginosa 11 (13) & 32 & 2 & 0.09 \\
\hline VIM-2 & P. aeruginosa 6 (14) & 128 & 16 & 0.38 \\
\hline VIM-2 & P. aeruginosa 11 (14) & 128 & 32 & 0.75 \\
\hline VIM-2 & P. aeruginosa 12 (14) & 128 & 32 & 0.75 \\
\hline VIM-2 & P. aeruginosa 13 (14) & 32 & 16 & 0.75 \\
\hline VIM-2 & P. aeruginosa 14 (14) & 64 & 2 & 0.06 \\
\hline VIM-2 & P. aeruginosa 15 (14) & 64 & 16 & 0.5 \\
\hline VIM-2 & P. aeruginosa 16 (14) & 64 & 16 & 0.5 \\
\hline VIM-2 & P. aeruginosa 27 (14) & 64 & 16 & 0.5 \\
\hline VIM-4 & E. cloacae 102 & 32 & 4 & 0.19 \\
\hline VIM-4 & E. coli 98 & 16 & 2 & 0.16 \\
\hline VIM-4 & K. oxytoca 95 & 32 & 8 & 0.38 \\
\hline VIM-4 & K. oxytoca 126 & 32 & 4 & 0.19 \\
\hline VIM-4 & K. pneumoniae 101 & 32 & 2 & 0.09 \\
\hline VIM-4 & K. pneumoniae 120 & 16 & 2 & 0.25 \\
\hline VIM-4 & K. pneumoniae 128 & 4 & 1 & 0.27 \\
\hline VIM-4 & К. pneumoniae 196 & 16 & 1 & 0.08 \\
\hline
\end{tabular}

larvae for $P$. aeruginosa GR57, E. coli 98 and K. pneumoniae respectively.

In treatment assays, survival of ML302F (4.8 $\left.\mathrm{mg} \mathrm{kg} \mathrm{kg}^{-1}\right)$ treated larvae was similar to those given PBS for the treatment of PA GR57, EC 98 and KP 120 infections. Monotherapy with MEM (0.6 $\mathrm{mg} \mathrm{kg} \mathrm{kg}^{-1}$ ) was superior to either PBS and ML302F ( $P<0.0001)$, but the \% survival was only 44\%, 23\% and 69\% versus strains EC 98, PA GR57 and KP 120, respectively. The addition of ML302F to MEM significantly improved the survival of infected larva $(P<0.0001)$ compared to MEM alone, with survival rates of 69\% (EC 98), 81\% (PA GR57) and 98\% (KP 120) at 96 (Fig. 2) and a relative risk for MEMML302F versus MEM of $0.64,0.28$ and 0.70 for these isolates.

\section{Experimental}

\section{Materials}

Bacterial strains were from the collection of clinical isolates held at Queen Mary University London. Species identification was by analysis of MADLI-TOF mass spectrometry profiles (Bruker) and MBL detection by PCR and sequencing of the $b l a_{\mathrm{VIM}}$ gene Meropenem trihydrate was purchased from Ranbaxy (UK) Limited (London, UK). ML302F was produced within the Chemical Research Laboratories, Oxford University 


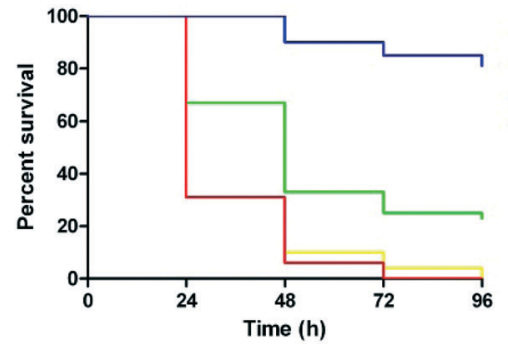

1. PBS

+ MEM

$\perp \mathrm{ML} 302 \mathrm{~F}$

- MEM + ML302F (1:8)

$4 \times 10^{1} \mathrm{CFU} /$ Larvae

inoculum

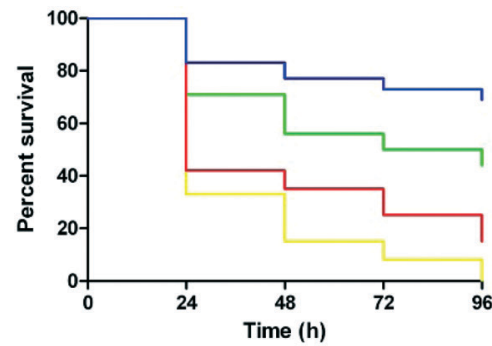

- PBS

$\perp$ MEM

+ ML302F

- MEM + ML302F (1:8)

$3 \times 10^{6} \mathrm{CFU} /$ Larvae

inoculum

b

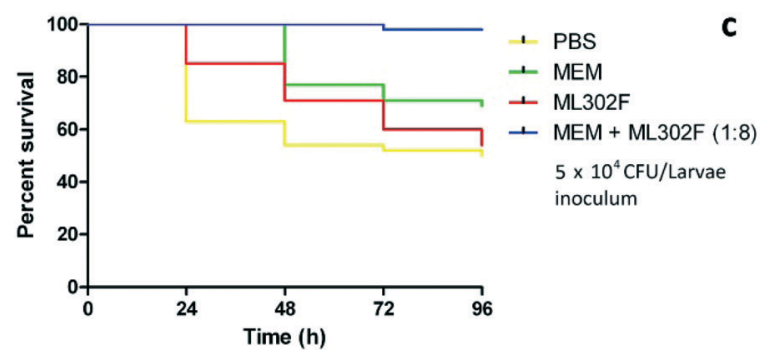

Fig. 2 Treatment assays using meropenem (MEM), ML302F and a 1: $8\left(\mathrm{mg} \mathrm{kg}^{-1}\right)$ combination of MEM + ML302F versus VIM-2 producing Pseudomonas aeruginosa isolate GR57 (a) VIM-4 producing Escherichia coli 98 (b) and Klebsiella pneumoniae 120 (c) over 96 h in Galleria mellonella.

(Oxford, UK). All media were purchased from SigmaAldrich (Dorset, UK).

\section{Minimum inhibitory concentrations}

Minimum inhibitory concentrations (MICs) of MEM (0-64 $\left.\mathrm{mg} \mathrm{L}^{-1}\right)$ and ML302F (0-32 $\left.\mathrm{mg} \mathrm{L}^{-1}\right)$ were determined by broth microtiter dilution (BMD) in Mueller-Hinton 2 cationadjusted broth with a bacterial inocula of $10^{5}$ colony forming units (CFU) per well in accordance with Clinical Laboratory Standards Institute (CLSI) guidance. Initial screening assays were performed using eight Gram-negative isolates including P. aeruginosa (VIM-2), Escherichia coli (VIM-4), Klebsiella pneumoniae (VIM-4) and Providencia stuartii (VIM-1). K. pneumoniae NCTC 9633 and P. aeruginosa ATCC 27853 were used as MEM susceptible type strains to control BMD MIC determinations.

The activity of ML302F in combination with MEM was assessed in checkerboard assays with fractional inhibitory concentration index (FICI) and MEM:ML302F ratio used to quantify interactions. ${ }^{10}$ A FICI (MIC of MEM/ML302F + MIC ML302F/MEM) of $\leq 0.5$ was defined as synergy, a FICI of 0.5 to 4.0 as indifferent or additive, and values of $>4.0$ as antagonistic. MICs for the MEM/ML302F combination strains studied were determined at fixed MEM:ML302F ratios of $1: 4$ and $1: 8$ by BMD.

\section{Toxicity assays}

The in vivo toxicity of ML302F was assessed in the Galleria mellonella wax moth larvae model using a simple live dead ranking. ${ }^{11}$ Increasing doubling concentrations of ML302F (0 to $80 \mathrm{mg} \mathrm{kg}^{-1}$ ) were administered in $10 \mu \mathrm{l}$ injections directly into the hemocoel of ten larvae before incubation at $37{ }^{\circ} \mathrm{C}$ for $96 \mathrm{~h}$. Larvae were scored for mellonisation and movement every $24 \mathrm{~h}$, as live or dead. Larvae for all G. mellonella assays were purchased from Livefood UK Ltd (Somerset, UK).

\section{G. mellonella virulence assay}

The bacterial inocula required for staggered killing (50\% lethal dose $\left.\left[\mathrm{LD}_{50}\right]\right)$ of $\mathrm{G}$. mellonella by VIM producing isolates over $96 \mathrm{~h}$ was determined using 10 larvae inoculated with overnight cultures of E. coli EC98, K. pneumoniae KP120 and $P$. aeruginosa PA GR57. Tenfold serial dilutions were made from washed cultures, diluted in PBS and administered in 10 $\mu \mathrm{l}$ injections at final concentrations of $10^{1}-10^{6} \mathrm{CFU}$ per larvae and incubated at $37^{\circ} \mathrm{C}$ for $96 \mathrm{~h}$. Larvae were scored as live or dead every $24 \mathrm{~h}$.

\section{MEM/ML302F treatment assays}

The activity of MEM and ML302F alone and in combination were assessed using $16 \mathrm{G}$. mellonella larva as previously described. ${ }^{12}$ Larvae were inoculated with $P$. aeruginosa GR57, E. coli EC98 and K. pneumoniae KP120. In combination therapies, a fixed ratio (1:8) of MEM:ML302F containing $0.6 \mathrm{mg}$ $\mathrm{kg}^{-1}$ of $\mathrm{MEM}^{8}$ and $4.8 \mathrm{mg} \mathrm{kg}{ }^{-1}$ of ML302F was administered in $10 \mu \mathrm{L}$ injections to infected larvae. PBS injections $(10 \mu \mathrm{L})$ were used as both controls for no antimicrobial therapy and inoculation injury.

Larvae were scored for survival over $96 \mathrm{~h}$ and kill curves analysed by the log-rank test for trend. Relative risk (RR) of survival between the different treatment arms was calculated using Fisher's exact test with, two-tailed $P$-values of $<0.05$ considered statistically significant.

\section{Conclusions}

In combination with MEM at a 1:8 ratio ML302F was able to restore susceptibility to MEM amongst VIM-producing carbapenem resistant Enterobacteriaceae (CRE) and was active in vivo in the G. mellonella larva invertebrate model of CRE infection. ML302F was active against several clinically relevant VIM variants. The combined results reveal that ML302F is a structurally interesting MBL inhibitor active in both cellular and animal models and should stimulate further work on the synthesis and testing of ML302F analogues. ML302F was derived from a rhodanine by hydrolysis. We are well aware that as a class the rhodanines have a somewhat chequered history with respect to their use as (potential) 


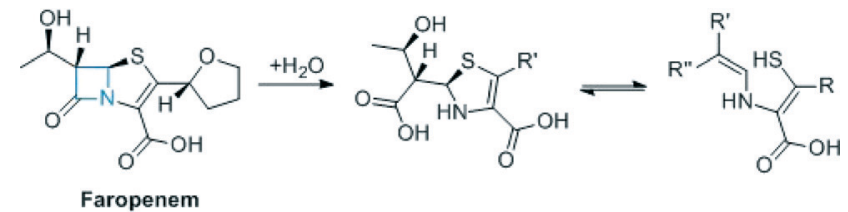

Fig. 3 Proposed mechanism for thioenol formation from faropenem.

pharmaceuticals. ${ }^{13,14}$ Furthermore, one may anticipate that the development of thioenols as pharmaceuticals might be difficult due to their reactivity (e.g. due to oxidation). However, as is well known the structures of many antibiotics do not follow recent 'normal' paradigms for drug structures. ${ }^{15}$ This is beautifully exemplified by the Class A serine $\beta$-lactamase inhibitors (clavulanic acid, tazobactam, sulbactam) that are widely used to extend penicillin and cephalosporin use. These are themselves $\beta$-lactams which act as 'mechanism based' inhibitors that undergo fragmentation on their target $\beta$-lactamases to produce ester linked species that are stable with respect to hydrolysis. ${ }^{15}$ The fact that rhodanines can undergo hydrolysis (whether enzyme mediated or not in the case of ML302/ML302F) to produce potent thioenol inhibitors raises the question as to whether mechanism based MBL inhibitors that are $\beta$-lactams which produce thioenol inhibitors can be generated. In this regard the development of modified carbapenems (one carbapenem, faropenem is in clinical use) ${ }^{16}$ (Fig. 3) which have the potential to undergo hydrolysis to produce thioenols that are MBL inhibitors may be of interest.

\section{Acknowledgements}

We would like to gratefully acknowledge Public Health England (Colindale, UK) and the General Hospital of Chest Diseases "Sotiria" (Athens, Greece) for supplying a number of antimicrobial-resistant isolates. We thank the Medical Research Council MR/L007665/1 grant for support of J. B. and C. J. S.

\section{Notes and references}

1 K. Bush, Ann. N.Y. Acad. Sci., 2013, 1277, 84-90.

2 A. M. Drawz, K. M. Papp-Wallace and R. A. Bonomo, Antimicrob. Agents Chemother., 2014, 58(4), 1835-1846.

3 W. Fast and L. D. Sutton, Biochim. Biophys. Acta, 2013, 1834(8), 1648-1659.

4 T. Spicer, D. Minond, I. Enogieru, S. A. Saldanha, C. Allais, Q. Liu, B. A. Mercer, W. R. Roush and P. Hodder, Probe Reports from the NIH Molecular Libraries Program (US National Center for Biotechnology Information), 2010.

5 J. Brem, S. S. van Berkel, W. S. Aik, A. M. Rydzik, M. B. Avison, I. Pettinati, K. D. Umland, A. Kawamura, J. Spencer, T. D. W. Claridge, M. A. McDonough and C. J. Schofield, Nat. Chem., 2015, 6, 1084-1090.

6 H. Ciesielczuk, J. Betts, L. Phee, M. Doumith, R. Hope, N. Woodford and D. W. Wareham, Virulence, 2015, 6, 145-151.

7 J. W.Betts, L. M. Phee, M. Hornsey, N. Woodford and D. W. Wareham, Antimicrob. Agents Chemother., 2014, 58, 3541-3546.

8 L. Hill, N. Veli and P. J. Coote, Int. J. Antimicrob. Agents, 2014, 52, 254-261.

9 A. Y. Peleg, S. Jara, D. Monga, G. M. Eliopoulos, R. C. Moellering and E. Mylonakis, Antimicrob. Agents Chemother., 2009, 53, 2605-2609.

10 M. J. Hall, R. F. Middleton and D. Westmacott, J. Antimicrob. Chemother., 1983, 11, 427-433.

11 A. P. Desbois and P. J. Coote, J. Antimicrob. Chemother., 2012, 66, 1785-1790.

12 M. Hornsey and D. W. Wareham, Antimicrob. Agents Chemother., 2011, 55, 3534-3537.

13 T. Mendgen, C. Steuer and C. D. Klein, J. Med. Chem., 2012, 55, 743-753.

14 M. A. T. Blaskovich, J. Zuegg, A. G. Elliot and M. A. Cooper, ACS Infect. Dis., 2015, 1, 285-287.

15 C. Bebrone, P. Lassaux, L. Vercheval, J. S. Sohier, A. Jehaes, E. Sauvage and M. Galleni, Drugs, 2010, 70, 651-679.

16 S. Hazra, S. G. Kurz, K. Wolff, L. Nguyen, R. A. Bonomo and J. S. Blanchard, Biochemistry, 2015, 54, 5657-5664. 